\title{
Involvement of UTR-dependent gene expression in the maintenance of cancer stem cell like phenotypes
}

\author{
MOTOAKI YASUDA $^{1}$, TOMOYUKI HATANAKA ${ }^{1}$, HIROKI SHIRATO ${ }^{2}$ and TAKESHI NISHIOKA ${ }^{3}$ \\ ${ }^{1}$ Department of Oral Pathobiology, Graduate School of Dental Medicine, Hokkaido University, Sapporo, Hokkaido 060-8586; \\ ${ }^{2}$ Department of Radiology and Nuclear Medicine, Graduate School of Medicine, Hokkaido University, Sapporo, \\ Hokkaido 060-8638; ${ }^{3}$ Department of Biological Sciences, Graduate School of Science, Hokkaido University, \\ Sapporo, Hokkaido 060-0812, Japan
}

Received October 18, 2014; Accepted August 5, 2015

DOI: $10.3892 / 01.2015 .3688$

\begin{abstract}
The present study demonstrated the acquisition of additional malignant characteristics in irradiated mouse fibrosarcoma cells compared with the parent cells. Several reporter assays indicated that hypoxia-inducible factor (HIF)- $1 \alpha$, activator protein-1 and Ets-dependent transcription were activated in irradiated cells. The cis-elements in the 5'-untranslated region (UTR) of these transcription factors plays a major role in their expression in surviving irradiated cancer cells. By contrast, there were no evident differences between the 3'-UTR-dependent repression demonstrated by parent cells and irradiated cells. A small population of parental fibrosarcoma cells was also found to exhibit the same enhanced 5'-UTR-dependent HIF-1 $\alpha$ expression as that demonstrated by irradiated cells. These observations may indicate that high-dose X-ray irradiation affects the majority of proliferating cancer cells, but not the cancer stem cells (CSCs), and an increased CSC population may explain the progressive phenotypes of the irradiated cells. It appears likely that the transcription factors that maintain stemness are regulated by the same 5'-UTR-dependent mechanism.
\end{abstract}

\section{Introduction}

Numerous studies have reported that the repopulation of malignant cells, such as through radiation-induced cancer progression, limits the effectiveness of radiation therapy (1-5).

Correspondence to: Dr Motoaki Yasuda, Department of Oral Pathobiology, Graduate School of Dental Medicine, Hokkaido University, Kita 13 Nishi 7, Kita-ku, Sapporo, Hokkaido 060-8586, Japan E-mail: moyasuda@den.hokudai.ac.jp

Dr Takeshi Nishioka, Department of Biological Sciences, Graduate School of Science, Hokkaido University, Kita 12 Nishi 5, Kita-ku, Sapporo, Hokkaido 060-0812, Japan

E-mail: trout@hs.hokudai.ac.jp

Key words: hypoxia-inducible factor- $1 \alpha$, Ets2, c-fos, repopulation, radio-resistance, untranslated region, cancer stem cell
Malaise et al (6) first revealed that the regrowth of irradiated transplantable mouse fibrosarcoma cells was faster compared with the regrowth of non-irradiated cells. Withers et al (4) performed a retrospective analysis and concluded that the repopulation of squamous cell carcinoma of the head and neck accelerated following a lag period of $\sim 4$ weeks subsequent to the initiation of radiotherapy. One possible interpretation of this study is that the protracted treatment schedule may permit a constant rate of repopulation throughout the administration of conventional fractionated radiotherapy. However, the molecular basis of the repopulation phenomenon remains unclear.

Over the previous decade, accumulated data have demonstrated that the 5' and 3'-untranslated regions (UTRs) play important roles in the expression of several genes, particularly in the post-transcriptional state (7-9). A previous study has demonstrated the reciprocal regulation of hypoxia-inducible factor (HIF)-1 $\alpha$ expression in a 5'- and 3'-UTR-dependent fashion (10). This previous observation also suggested a possible correlation between UTR-dependent regulation of HIF-1 $\alpha$ and cancer malignancy.

The present study aimed to demonstrate the enhanced expression of several transcription factors involved in cancer stem cell maintenance through a 5'-UTR-dependent mechanism in irradiated mouse fibrosarcoma cells.

\section{Materials and methods}

Plasmids and reagents. Luciferase reporter plasmids (PathDetect pAP-1and PathDetect p53) were purchased from Agilent Technologies (Santa Clara, CA, USA). Hypoxia responsible elements, Ets binding sequences and 536 bp (1457-1992 bp) of the human matrix metalloproteinase 1 (MMP1) enhancer sequence (GenBank ID, AY769434.1) were cloned into a pGL3 basic vector (Promega, Madison, WI, USA). Full length HIF-1 $\alpha$, which consisted of 294 bp of the 5'-UTR, 2,481 bp of the coding region and 1,195 bp of the 3'-UTR of HIF-1 $\alpha$, was cloned into the pcDNA3 vector (Invitrogen, Carlsbad, CA, USA) and designated as pcDNA HIF-5C3. pcDNA HIF-5C lacked the 3'-UTR of HIF-1 $\alpha$ and pcDNA HIF-C3 lacked the 5 '-UTR of HIF-1 $\alpha$. pcDNA HIF-C contained only the coding region for HIF-1 $\alpha$ (10). The full-length expression plasmids for 
mouse c-fos and Ets2 were purchased from Origene (Rockville, MD, USA). Four 3'-UTR sequences, consisting of the 3'-UTR HIF-1 $\alpha$, cMyc, Ets2 and c-fos sequences, were cloned downstream of the luciferase gene of the pmir GLO vector (Promega). Cobalt(II) chloride hexahydrate and G418 disulfate salt were purchased from Sigma-Aldrich (St. Louis, MO, USA).

Cell culture and cell cloning. The transplantable fibrosarcoma QRsP cell line, which was established by the present authors and is described elsewhere $(11,12)$ and NIH3T3 cells (RCB0150: Riken, Tsukuba, Japan), was cultured with Dulbecco's modified Eagle's medium (DMEM) containing $8 \%$ fetal bovine serum (FBS). In total, $1 \times 10^{6} \mathrm{QRsP}$ cells were irradiated at a dose of 10 Gy using a LINAC system (Toshiba, Tokyo, Japan). Trypsinized QRsP cells were seeded onto $10 \mathrm{~cm}$ dishes $1 \mathrm{~h}$ subsequent to irradiation and cultured for 14 days. Well-demarcated colonies were trypsinized using a cloning cylinder and grown in DMEM for 14 days. Subsequently, 6 independent cell lines were established and classified as QRsPIR-1 to QRsPIR-6. The parental QRsP cells and the QRsPIR-1 and QRsPIR-2 cell lines were implanted subcutaneously into the dorsal area of 6 -week-old female c57bl/6 mice (CLEA Japan, Inc., Tokyo, Japan). The cells were recovered from the tumor mass of each animal 28 days subsequent to implantation and designated as QRsPV, QRsPV-IR1 and QRsPV-IR2. All cells were stored at $-80^{\circ} \mathrm{C}$ for additional analysis. In order to mimic hypoxic conditions, the cells were exposed to $200 \mu \mathrm{M} \mathrm{CoCl}_{2}$ diluted in DMEM, for $16 \mathrm{~h}$. The animal experiments were strictly compliant with the animal care guidelines of Hokkaido University (Sapporo, Hokkaido, Japan).

Colony assay. QRsPV cells were transfected with pcDNA HIF-5C, and $24 \mathrm{~h}$ later, $1 \times 10^{6}$ cells were irradiated at a dose of 4 , 8 or 10 Gy using the LINAC system (Toshiba, Tokyo, Japan). In total, $1 \times 10^{6}$ trypsinized cells were seeded onto $60-\mathrm{mm}$ culture dishes $1 \mathrm{~h}$ subsequent to irradiation, and $1 \times 10^{4}$ non-irradiated cells that had been transfected with the pcDNA3 vector were also seeded onto $60-\mathrm{mm}$ culture dishes to obtain the plating efficiency. The plating efficiency was calculated as the percentage of cells seeded that grow into colonies under G418 selection conditions. The cells were cultured for 2 weeks with DMEM containing G418 at a final concentration of $1 \mathrm{mg} / \mathrm{ml}$, followed by methanol fixation and Giemsa staining (2\% Giemsa's solution: Merck-Millipore, Darmstadt, Germany).

Histopathological examination. Semi-confluent QRsPV or QRsPV-IR2 cells were trypsinized and resuspended with phosphate-buffered saline (PBS). In total, 1 $110^{4}$ PBS-suspended cells were injected subcutaneously into the dorsal region of 6-week-old female c57bl/6 mice, with 3 mice per group. On day 28 , all animals were sacrificed and the tumor masses were dissected. The tumor tissues were fixed in 10\% formaldehyde, with occasional de-calcification, and then embedded in paraffin, according to routine pathological procedure. The specimens were sliced into $5-\mu \mathrm{m}$ thick sections and stained with hematoxylin and eosin.

Western blotting. The cells were lysed in a buffer containing $250 \mathrm{mM} \mathrm{NaCl}, 50 \mathrm{mM}$ HEPES (pH 7.0) and $0.1 \%$ Nonidet
P-40 with a protease inhibitor cocktail (Sigma-Aldrich, St. Louis, MO, USA). The samples were then subjected to western blotting using anti-human HIF-1 $\alpha$ mouse monoclonal antibody was purchased from BD Biosciences (Cat no. 610959, Sparks, MD, USA) and anti-Von Hippel-Lindau (VHL) monoclonal antibody was purchased from Santa Cruz Biotechnology (Cat no. sc-17780, Dallas, TX, USA). Both primary antibodies were used at dilutions of 1:1,000. Horseradish Peroxidase conjugated donkey anti-mouse secondary antibody was purchased from Jackson Immunoresearch (West Grove, PA, USA) and was used at dilutions of 1:2,000. Amersham ECL reagents were purchased from GE Healthcare Bio-Sciences (Pittsburgh, PA, USA).

Reporter assay. The QRsPV cells were plated at a density of $1 \times 10^{5}$ cells per well in 24-well plates on the day prior to transfection. The cells were transiently transfected with Lipofectamine 2000 reagent (Invitrogen, Carlsbad, CA, USA) containing $75 \mathrm{ng}$ of luciferase reporters and $7.5 \mathrm{ng}$ of pGL4.72 luciferase reporter vector (Promega) and $300 \mathrm{ng}$ of a series of HIF-1 $\alpha$ expression plasmids (Fig. 2A). The cell lysates were subjected to luciferase assays using a dual-luciferase reporter assay system (Promega) $24 \mathrm{~h}$ subsequent to transfection, according to the manufacturer's instructions.

Statistical analysis. Statistical differences were analyzed using Student's $t$-test. $\mathrm{P} \leq 0.05$ was considered to indicate a statistically significant difference.

\section{Results}

Cloning of repopulated cancer cells. Accumulated data have suggested that accelerated repopulation of irradiated cancer cells occurs 3 weeks subsequent to irradiation $(4,5)$. To analyze this phenomenon, irradiated or non-irradiated QRsP cells were injected into the dorsal region of $\mathrm{c} 57 \mathrm{bl} / 6$ mice. After 28 days, the tumor masses were collected for tissue culture and designated as QRsPV, QRsPV-IR1 and QRsPV-IR2 tumors. The cells were re-inoculated into mice, with 3 mice each in the QRsPV, QRsPV-IR1 and QRsPV-IR2 groups, for 28 days. During the incubation period, 1 out of 3 animals inoculated with QRsPV-IR2 cells demonstrated severe leg paralysis. As indicated in Fig. 1A, the QRsPV-IR2 cells demonstrated an infiltrative morphology, whereas QRsPV cells revealed well-demarcated expansive growth, without evident infiltration. Therefore, the QRsPV-IR2 cells were utilized as a model for an irradiated progressed cell line in subsequent experiments. In the following experiment, several reporter constructs, consisting of HIF-1 $\alpha$, p53, AP-1 and Ets consensus sequence-containing luciferase reporters, were introduced into QRsPV and QRsPV-IR2 cells. In the QRsPV-IR2 cells, HIF-1 $\alpha$, AP-1 and Ets-dependent endogenous transcriptional reactions were significantly increased $(\mathrm{P}=0.00036$, $\mathrm{P}=0.027$ and $\mathrm{P}=0.017$, respectively) compared with the $\mathrm{QRsPV}$ cells, whereas p53-dependent transcription was severely decreased ( $\mathrm{P}=0.017)$ (Fig. 1B).

HIF-1 $\alpha$ expression was activated in irradiated cells. To confirm the 5'- and 3'-UTR dependent expression regulation in the QRsPV cells, HIF-1 $\alpha$ plasmids (Fig. 2A; HIF5C3, 5C, C 
A

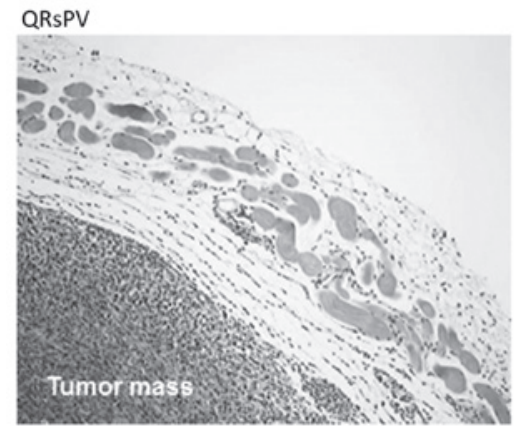

C

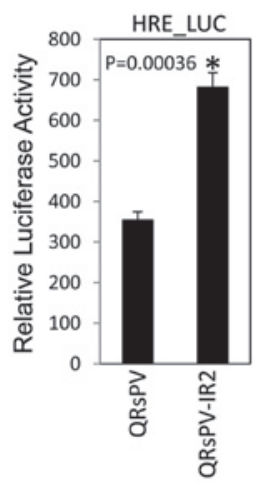

B

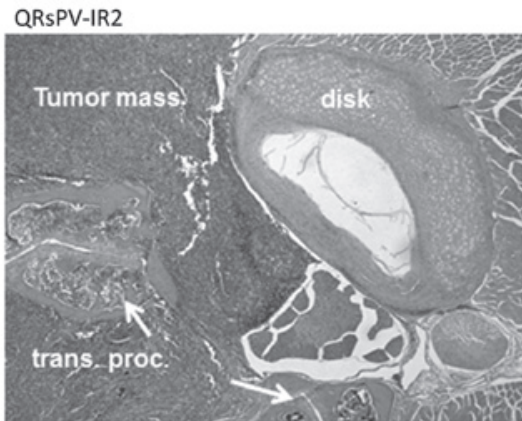

Figure 1. Invasive phenotypes of radiation-treated cells. Histopathological images of mouse tissues obtained from animals that were implanted with (A) QRsPV or (B) QRsPV-IR2 cells. (A) Well-defined borders between the dorsal muscles and tumor masses were observed in QRsPV lesions. (B) Aggressive invasion of QRsPV-IR2 cells into the vertebral region was also identified, and the intra-spinal space was occupied by a tumor mass. (C) The indicated luciferase reporters were transfected into QRsPV and QRsPV-IR2 cells. Error bars represent the standard deviations for triplicate wells in a single experiment, and the data are representative of three independent experiments. "P $\leq 0.05$. Disk, intervertebral disk; trans. proc., transverse process; HRE, hypoxia response element; LUC, luciferase; AP-1, activator protein-1.

and C3) were transfected and cell lysates underwent western blot analysis. As indicated in Fig. 2B, cells transfected with HIF-5C 3 and HIF-5C expressed an increased amount of HIF-1 $\alpha$ protein compared with HIF-C. Low expression of HIF-1 $\alpha$ was demonstrated in QRsPV cells (Fig. 2B). In the subsequent experiment, QRsPV and QRsPV-IR2 cells were transfected with several HIF-1 $\alpha$ plasmids combined with an HRE luciferase reporter and a Renilla luciferase plasmid. Accelerated 5'-UTR-dependent translation of HIF-1 $\alpha$ is evident in irradiated QRsPV-IR2 cells (Fig. 2C). Increased HIF-1 $\alpha$ expression was observed in QRsPV-IR2 cells transfected with HIF-5C compared with the cells transfected with HIF-C $(\mathrm{P}=0.025)$. The difference in HIF-1 $\alpha$ expression was not significant between the HIF-5C-transfected and HIF-C-transfected QRsPV cells $(\mathrm{P}=0.065)$. These observations encouraged the investigation of the accumulation of HIF-1 $\alpha$ under hypoxia-mimicking conditions in QRsPV and QRsP-IR2 cells. As demonstrated in Fig. 2D, $>2$ fold HIF-1 $\alpha$-dependent luciferase activity was detected in QRsPV-IR2 cells under hypoxia-mimicking conditions, which was a significant difference $(\mathrm{P}=0.025)$.

Enhanced AP-1 and Ets-associated gene expression in irradiated cells. Since activated AP-1 and Ets-dependent transcription was observed in QRsPV-IR2 cells, the UTR-dependent expressional regulation of c-fos and Ets 2 was investigated. As described in Fig. 3A, the two mRNA sequences contained relatively long 5'- and 3'-UTRs. Typical AU-rich (ARE) elements were identified in the two mRNA sequences, with 3 AREs in c-fos and 4 AREs in Ets2 (Fig. 3A). The two plasmids were transfected together with a MMP1-luciferase reporter, as AP-1 and Ets binding sites are available on this construct, and a Renilla plasmid into the QRsPV and QRsPV-IR2 cells. Fig. 3A reveals the significantly enhanced luciferase activity associated with c-fos $(\mathrm{P}=0.002)$ and Ets2 $(\mathrm{P}=0.038)$ expression in QRsPV-IR2 cells compared with QRsPV cells. These observations prompted the confirmation of whether the 3'-UTR-dependent repressive system is activated in irradiated cells. Several 3'-UTR sequences were cloned downstream of luciferase sequences in pmir GLO vectors (Fig. 3B). All constructs were introduced into NIH3T3 negative control, QRsPV and QRsPV-IR2 cells. As indicated in Fig. 3B, there was a significant repressive effect via HIF1 $\alpha$ 3'UTR in NIH3T3 $(\mathrm{P}=0.03)$, but not in QRsPV $(\mathrm{P}=0.14)$ and QRsP-IR2 cells $(\mathrm{P}=0.10)$. These results indicate that 3 '-UTR-dependent regulatory machinery may not be involved in the enhanced gene expression in irradiated cells.

Selective effect of $X$-ray irradiation. Since cancer tissues consist of a heterogeneous cell population $(13,14)$, the majority of which are proliferating cancer cells and CSCs, it is possible that the high-dose X-ray irradiation affects only the proliferating cancer cells under the present experimental conditions. Therefore, certain components of the QRsP parent cell population, in particular the CSC-like population of QRsP cells, should exhibit enhanced 5'-UTR dependent HIF-1 $\alpha$ expression, as observed in QRsPV-IR2 cells. To address this issue, 24 independent single-cell-derived QRsPV cells, designated A through $\mathrm{X}$, were cloned and reporter assays were performed. 
A

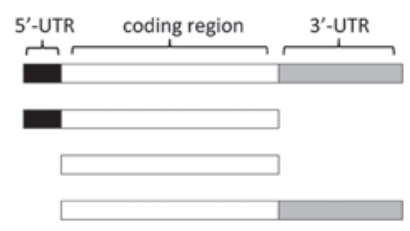

C

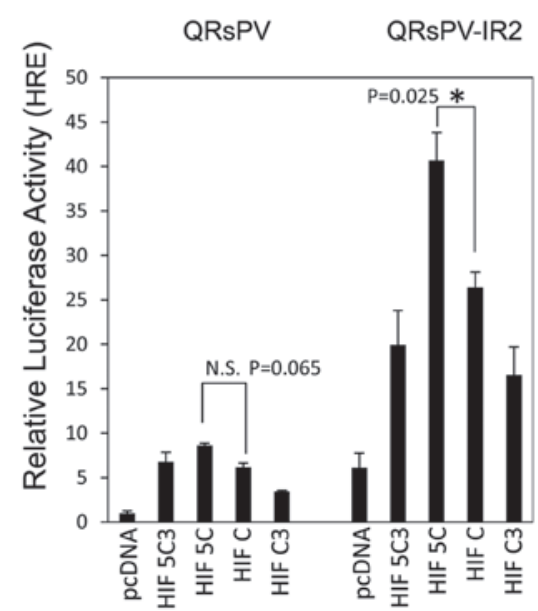

B

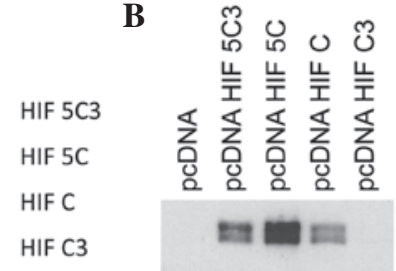

IB: anti HIF-1 $1 \alpha$

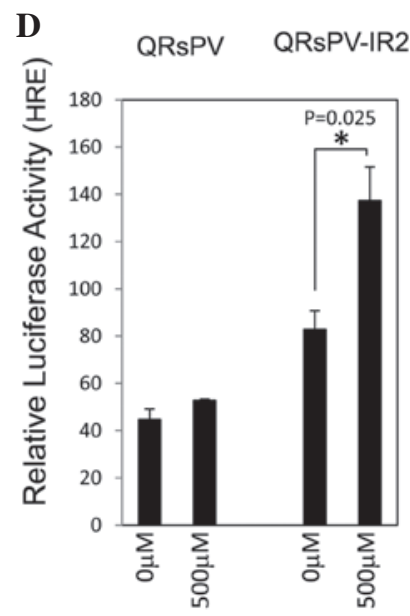

Figure 2. HIF-1 $\alpha$ expression in QRsPV and QRsPV-IR2 cells. (A) Schematic illustrations of HIF-1 $\alpha$ constructs are indicated. All asterisks indicate AU-rich elements. (B) Results of western blot analysis performed using an anti-HIF-1 $\alpha$ monoclonal antibody. The QRsPV cells were transfected with the indicated plasmids. (C) Luciferase reporter assay for HIF-1 $\alpha$. An accelerated expression of HIF-1 $\alpha$ was observed in HIF 5C-transfected QRsPV cells, but not in QRsPV cells. The relative luciferase activity is reported. An accelerated 5'-UTR-dependent translation was observed in QRsPV-IR2 cells. The error bars represent the standard deviation_for triplicate wells in a single experiment, and the data are representative of three independent experiments. (D) HIF-1 $\alpha$ expression in the hypoxia-mimicking condition. The QRsPV and QRsPV-IR2 cells were transfected with HRE-luciferase reporters. In total, a final concentration of $500 \mu \mathrm{M}$ cobalt chloride was added to the culture medium $24 \mathrm{~h}$ subsequent to transfection, and the cells were incubated for an additional $6 \mathrm{~h}$. The error bars represent standard deviations for triplicate wells in a single experiment, and the data are representative of three independent experiments. ${ }^{*} \leq 0.05$. HIF-1 $\alpha$, hypoxia-inducible factor-1 $\alpha$; IB, immunoblot; N.S., not significant; UTR, untranslated region.

As indicated in Fig. 4A, 2 out of the 24 clones (A and M1) exhibited similar luciferase activity to that exhibited by the QRsPV-IR2 cells. It should be noted that there was no evident difference between the endogenous expression levels of VHL in these cell lines (Fig. 4B).

These results encouraged the investigation of whether the cells expressing high HIF-1 $\alpha$ levels were capable of surviving the lethal damage of ionizing irradiation. Colony assays were performed using QRsPV cells transfected with HIF-1 $\alpha$ or a control vector. The transfected cells were irradiated at a dose of 4, 8 or $10 \mathrm{~Gy}$, followed by selection for 10 days in G418 and Giemsa staining. As indicated in Fig. 4C, HIF-1 $\alpha$-transfected cells revealed a radio-resistant phenotype compared with the control at radiation doses of 4,8 and $10 \mathrm{~Gy}(\mathrm{P}=0.046$, $\mathrm{P}=0.025$ and $\mathrm{P}=0.044$, respectively). The mean size of colonies consisting of HIF-1 $\alpha$-transfected cells was increased compared with control transfectants (data not shown). It is likely that the total number of cells surviving irradiation, rather than the colony number, was increased by HIF-1 $\alpha$ overexpression.

\section{Discussion}

CSCs are considered to be responsible for the onset, self-renewal, mutation accumulation and metastasis of tumors. CSCs may exist as dormant cells within the primary tumor mass $(13,14)$, but they may transition between a dormant state and an actively proliferating state due to genotoxic damage caused by ionizing irradiation or chemotherapeutic treatment. If the genotoxic damage kills the majority of proliferating cancer cells, but not the CSCs, over the prolonged course of therapy, the CSCs may be activated and the percentage of CSCs in the residual tumor mass may be increased. The existence of a large number of CSCs in the primary lesion may explain the high frequency of relapse, distant metastasis and resistance to cancer therapy. These concepts demonstrate an extremely good fit for the conventional repopulation theory.

Previously, the reciprocal regulation of HIF-1 $\alpha$ by 5'- and 3'-UTR-dependent mechanisms and the possible correlation between 5' UTR-dependent translational regulation of HIF-1 $\alpha$ and tumor malignancies have been reported (10). In the present study, the cells that survived radiation, the QRsPV-IR2 cells, demonstrated a more aggressive phenotype compared with the non-irradiated QRsPV cells. The 5'-UTR-dependent translational activity of HIF-1 $\alpha$ was markedly increased in the QRsPVIR-2 cells, even under a normoxic environment. Increased endogenous HIF-1 $\alpha$ expression in the QRsPV-IR2 cells was also identified under hypoxia-mimicking conditions. Overall, these results suggest that the QRsPV-IR2 cells that survived radiation may express larger levels of HIF-1 $\alpha$, Ets2 and c-fos proteins and may exhibit a more aggressive phenotype compared with the parental cell line. It was also notable that a repression of p53 dependent transcriptional activity was observed in QRsPV-IR2 cells. Previous studies have reported the 

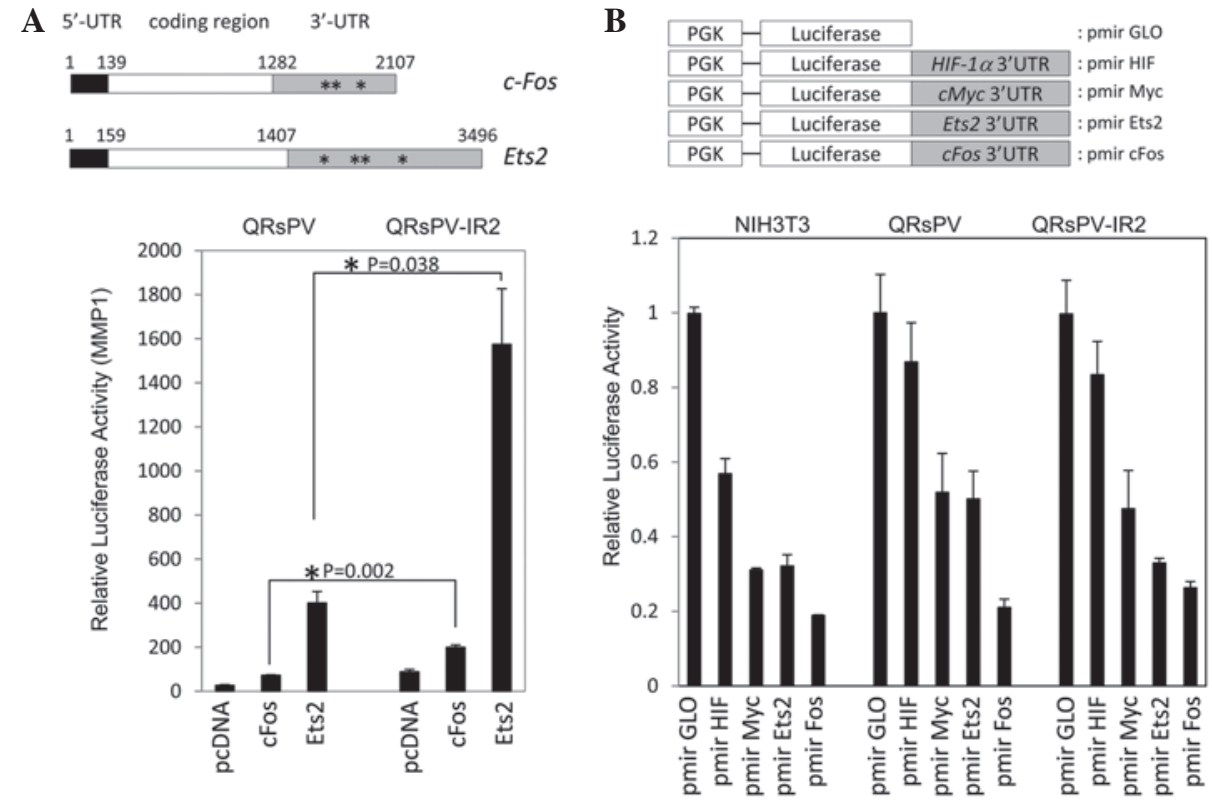

Figure 3. Enhanced expression of c-fos and Ets2. (A) Shematic illustrations of c-fos and Ets 2 constructs and results of reporter the assays are presented. An MMP1-luciferase reporter, containing activator protein-1 and Ets2 sites, was transfected together with a Renilla plasmid and the indicated plasmids. The error bars represent the SDs for triplicate wells in a single experiment, and the data are representative of three independent experiments. (B) Shematic illustrations of pmir GLO-based constructs and results of luciferase assays are indicated. A drastic repression of luciferase activity was identified in NIH3T3 cells. The QRsPV and QRsPV-IR2 cells demonstrated similar expression patterns. The average luciferase activities are reported, with the error bars representing the SDs for triplicate wells in a single experiment, and the data are representative of three independent experiments. "P $\leq 0.05$. MMP1, matrix metalloproteinase 1; SD, standard deviation; UTR, untranslated region.

A

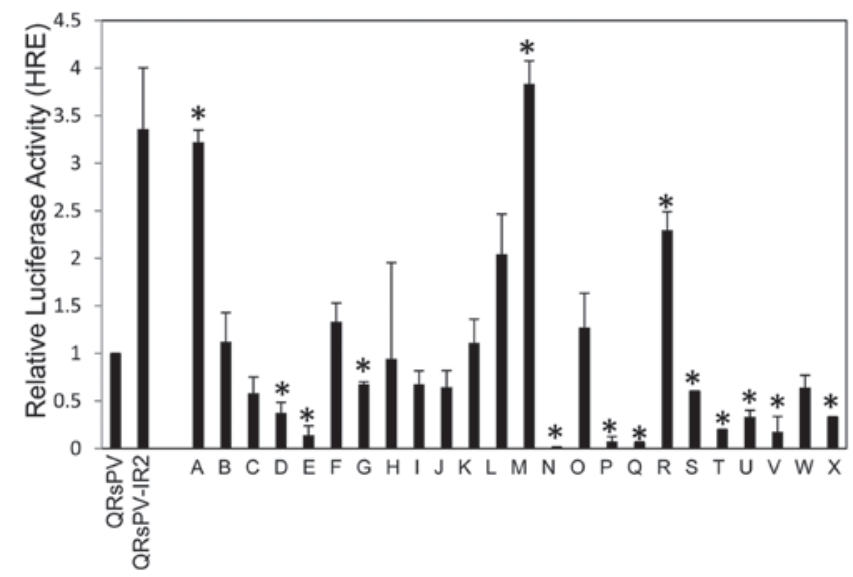

B

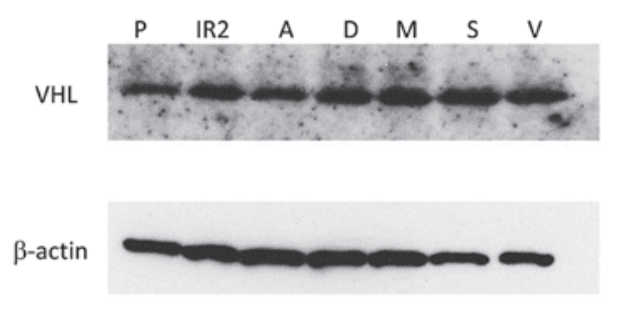

C

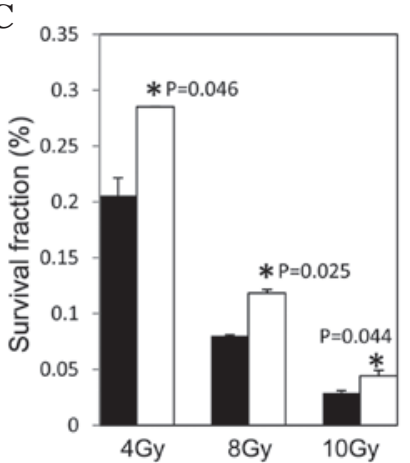

Figure 4. HIF-1 $\alpha$ expression and a radio-resistant phenotype in cancer stem cell-like cells. (A) Relative luciferase activities of HIF-5C-transfected cells are indicated. Cells designated A through X were single-cell derived clones of QRsPV cells. Only 2 clones demonstrated comparable luciferase activity to that exhibited by QRsPV-IR2 cells. The error bars represent standard deviations for triplicate wells in a single experiment. The data are representative of three independent experiments. (B) The results of immunoblot analysis performed using an anti-VHL monoclonal antibody and QRsPV-derived clones, as indicated. Comparable VHL protein expressions were observed. (C) Exogenous HIF-1 $\alpha$ expression induced the radio-resistant phenotype of cancer cells. The radio-resistant surviving fractions of the HIF-5C-transfected QRsPV cells (white bars) and control cells (black bars) are indicated. The error bars indicate the standard deviations for triplicate culture dishes, and the data are representative of two independent experiments. " $\mathrm{P} \leq 0.05$ was considered to indicate a statistically significant difference. HRE, hypoxia response element; HIF, hypoxia-inducible factor; VHL, Von Hippel-Lindau. 
involvement of cMyc, HIF-1 $\alpha$ and Ets2 transcription factors in CSC maintenance (15-17). By contrast, p53 has been regarded as the barrier to CSC formation $(15,18,19)$. It is likely that the expression pattern found in QRsPV-IR2 cells is suitable for maintaining CSC-like phenotypes.

It was also found in the present study that parental QRsPV cells contained a small population that exhibited the same phenotype as radiation-resistant CSC-like QRsPV-IR2 cells. Notably, exogenous expression of HIF-1 $\alpha$ was sufficient to activate the resistant phenotype of QRsPV cells against high-dose X-ray irradiation. These observations prompted the hypothesis that 10-Gy irradiation is lethal for the majority of proliferating QRsPV cells, but not for CSC-like QRsPV cells expressing an increased level of HIF-1 $\alpha$. It appears likely that the QRsPV-IR2 tumors contained a larger number of CSCs. Whole genomic sequencing using next generation sequencing techniques is required to eliminate the possibility that $\mathrm{X}$-irradiation induced the genomic mutations or genomic modification of cancer cells under the present experimental conditions.

A large number of transcription factors may contribute to the maintenance of the stemness of CSCs (15-17). It appears possible that several transcription factors that are involved in CSC maintenance, are regulated by the same mechanism in an UTR-dependent manner. If these CSC maintenance genes are regulated simultaneously by the same molecular mechanism, such a novel pathway may be a promising future target for the suppression of CSCs.

\section{Acknowledgements}

This study was supported by a Grant-in-Aid for Scientific Research (grant no. B24390285) provided by the Ministry of Education, Science, and Culture of Japan.

\section{References}

1. Allam A, Perez LA, Huang P, Taghian A, Azinovic I, Freeman J, Duffy M, Efird J and Suit HD: The effect of the overall treatment time of fractionated irradiation on the tumor control probability of a human soft tissue sarcoma xenograft in nude mice. Int J Radiat Oncol Biol Phys 32: 105-111, 1995.

2. Beck-Bornholdt HP, Omniczynski M, Theis E, Vogler H and Würschmidt F: Influence of treatment time on the response of rat rhabdomyosarcoma $\mathrm{R} 1 \mathrm{H}$ to fractionated irradiation. Acta Oncol 30: 57-63, 1991.
3. Begg AC, Hofland I and Kummermehr J: Tumour cell repopulation during fractionated radiotherapy: Correlation between flow cytometric and radiobiological data in three murine tumours. Eur J Cancer 27: 537-543, 1991.

4. Withers HR, Maciejewski B, Taylor JM and Hliniak A: Accelerated repopulation in head and neck cancer. Front Radiat Ther Oncol 22: 105-110, 1988.

5. Withers HR, Taylor JM and Maciejewski B: The hazard of accelerated tumor clonogen repopulation during radiotherapy. Acta Oncol 27: 131-146, 1988.

6. Malaise E and Tubiana M: Growth of the cells of an experimental irradiated fibrosarcoma in the $\mathrm{C} 3 \mathrm{H}$ mouse. $\mathrm{C} \mathrm{R}$ Acad Sci Hebd Seances Acad Sci D 263: 292-295, 1966 (In French).

7. Barreau C, Paillard L and Osborne HB: AU-rich elements and associated factors: Are there unifying principles? Nucleic Acids Res 33: 7138-7150, 2006.

8. Hsieh AC, Liu Y, Edlind MP, Ingolia NT, Janes MR, Sher A Shi EY, Stumpf CR, Christensen C, Bonham MJ, et al: The translational landscape of mTOR signalling steers cancer initiation and metastasis. Nature 485: 55-61, 2012.

9. Huntzinger E and Izaurralde E: Gene silencing by microRNAs: contributions of translational repression and mRNA decay. Nat Rev Genet 12: 99-110, 2011

10. Yasuda M, Hatanaka T, Shirato H and Nishioka T: Cell type-specific reciprocal regulation of HIF1A gene expression is dependent on 5'- and 3'-UTRs. Biochem Biophys Res Commun 447: 638-643, 2014.

11. Habelhah H, Okada F, Kobayashi M, Nakai K, Choi S, Hamada J, Moriuchi T, Kaya M, Yoshida K, Fujinaga K and Hosokawa M: Increased E1AF expression in mouse fibrosarcoma promotes metastasis through induction of MT1-MMP expression. Oncogene 18: 1771-1776, 1999.

12. Nishioka T, Miyai Y, Haga H, Kawabata K, Shirato H, Homma A, Shibata K and Yasuda M: Novel function of transcription factor ATF5: blockade of p53-dependent apoptosis induced by ionizing irradiation. Cell Struct Funct 34: 17-22, 2009.

13. Sagar J, Chaib B, Sales K, Winslet M and Seifalian A: Role of stem cells in cancer therapy and cancer stem cells: A review. Cancer Cell Int 7: 9, 2007.

14. Sales KM, Winslet MC and Seifalian AM: Stem cells and cancer: an overview. Stem Cell Rev 3: 249-255, 2007.

15. Akita H, Marquardt JU, Durkin ME, Kitade M, Seo D, Conner EA, Andersen JB, Factor VM and Thorgeirsson SS: MYC activates stem-like cell potential in hepatocarcinoma by a p53-dependent mechanism. Cancer Res 74: 5903-5913, 2014.

16. Keith B and Simon MC: Hypoxia-inducible factors, stem cells and cancer. Cell 129: 465-472, 2007.

17. Múnera J, Ceceña G, Jedlicka P, Wankell M and Oshima RG: Ets2 regulates colonic stem cells and sensitivity to tumorigenesis. Stem Cells 29: 430-439, 2011.

18. Aloni-Grinstein R, Shetzer Y, Kaufman T and Rotter V: p53: The barrier to cancer stem cell formation. FEBS Lett 588: 2580-2589, 2014.

19. Bonizzi G, Cicalese A, Insinga A and Pelicci PG: The emerging role of p53 in stem cells. Trends Mol Med 18: 6-12, 2012. 